\title{
Nonequilibrium Regulation of Interfacial Chemistry for Dissipative Supramolecular Assembly of Macroscopic Hydrogels
}

Ting Zhao, ${ }^{1}$ Yuyu E, ${ }^{1}$ Jiwei Cui, ${ }^{2}$ Jingcheng Hao, ${ }^{2}$ Xu Wang ${ }^{*}, 1,2$

${ }^{1}$ National Engineering Research Center for Colloidal Materials, School of Chemistry and Chemical Engineering, Shandong University, Jinan, Shandong 250100, P. R. China

${ }^{2}$ Key Laboratory of Colloid and Interface Chemistry of the Ministry of Education, School of Chemistry and Chemical Engineering, Shandong University, Jinan, Shandong 250100, P. R. China

*Corresponding author: wangxu@sdu.edu.cn 


\section{ABSTRACT:}

The nonequilibrium assembly in nature exists at the microscopic and macroscopic scales, and represents the most common way to regulate object motions by consuming chemical fuels. In artificial nonequilibrium systems, most of the work has focused on the microscopic dissipative assembly, whereas the investigation on nonequilibrium assembly of macroscopic building blocks is rarely reported. Here, we present an efficient strategy to dynamically mediate the interfacial chemistry of $\mathrm{pH}$-responsive polyelectrolyte hydrogels, thereby regulating their macroscopic nonequilibrium assembly. The driving force for the assembly is the transient electrostatic attraction, which is regulated by the biocatalytic feedback-driven temporal programming of the $\mathrm{pH}$ of the system. The dissipative assembly process can be controlled by adjusting the hydrogel parameters and fuel composition and it can be repeated by refueling the system. Most importantly, the ordered sewing of complementary hydrogels promotes the precise nonequilibrium supramolecular assembly to yield transient macroscopic supramolecular devices potentially useful for timed release. 


\section{INTRODUCTION}

The nonequilibrium assembly in nature is a common and essential process. ${ }^{1-5}$ The dissipative self-assembling systems always run at high energy states and maintain the active structures by consuming chemical fuels, which are the basis for living organisms to perform their complex functions. 4, 6,7 Diverse transient assembly processes exist in life forms on both micro and macroscales. ${ }^{8,9}$ Learning from nature, the dissipative assembly of synthetic molecules driven by chemical fuels was suggested in 2010 by Boekhoven et al. ${ }^{10}$ Now, scientists have successfully constructed diverse nonequilibrium systems by adopting various types of microscopic building blocks, such as small molecules, ${ }^{11-14}$ synthetic polymers, ${ }^{15,}{ }^{16}$ biomacromolecules, ${ }^{17,}, 18$ and nanoparticles. ${ }^{19-21}$ Recent work has also shown promise in controlling drug release behaviors ${ }^{22}$ and self-healing functions ${ }^{15,23-25}$ in nonequilibrium systems. Despite these achievements, current studies in this filed are still focusing on the micro level, and far too little attention has been paid to the macroscopic nonequilibrium assembly.

Macroscopic assembly refers to a process of clustering of building blocks larger than ten micrometers, ${ }^{26}$ commonly driven by long-ranged forces, ${ }^{27,}{ }^{28}$ covalent bonds, ${ }^{29}$ or supramolecular interactions. $^{30-33}$ With the rapid development of supramolecular chemistry, ${ }^{34}$ macroscopic supramolecular assembly has been intensively studied for a decade since the first example was presented by Harada et al. ${ }^{30}$ Up to now, the supramolecular assembly of macroscopic building blocks with different material textures, hardness and surface chemistries has been performed, ${ }^{35,36}$ which sheds new light on the possibility of using macroscopic assembly to fabricate biomedical 
materials. ${ }^{37}$ Simultaneously, the controlled dissociation of macroscopic assemblies is also important, and the programmed disassembly has been demonstrated to be useful in overcoming kinetically trapped assembly states for improving assembly accuracy. ${ }^{26}$ Current strategies to achieve macroscopic disassembly mainly rely on external stimuli such as acid, ${ }^{38}$ salt, $^{26}$ and heat triggers $^{39}$ to destroy the dynamic covalent bonds or supramolecular interactions at the interacting hydrogel interfaces. In principle, a more advanced method is to use preprogrammed reactions to regulate the nonequilibrium transient assembly (successive assembly and disassembly) of macroscopic building blocks; unfortunately, this method has yet to be experimentally verified. The nonequilibrium macroscopic assembly enables the self-regulated and temporal control of macroscopic supramolecular devices, which are of great interest in the fields of supramolecular chemistry and materials science.

Here, we designed a self-regulated and temporally controlled system to demonstrate that transient assembly of macroscopic materials can be performed by activating the nonequilibrium supramolecular interactions. The enzyme-entrapped, $\mathrm{pH}$-sensitive polyelectrolyte hydrogels are used as macroscopic building blocks that can achieve the nonequilibrium assembly in response to the addition of chemical fuels. The programmed assembly-disassembly cycle of the polyelectrolyte hydrogels is controlled by a switching between the electrostatic attraction and repulsion at the interacting hydrogel interfaces, which is essentially regulated by an enzyme-mediated competing process. Moreover, an ordered sewing/growing method is developed for the fabrication of hybrid polyelectrolyte hydrogels with modularized surface 
chemistry. The resulting hydrogels can form transient macroscopic supramolecular assemblies with controllable shapes and lifespans.

\section{EXPERIMENTAL SECTION}

Materials. Acrylamide (AAm), N,N'-methylenebis(acrylamide) (MBAAm), sodium p-styrenesulfonate (SSNa), 4-vinyl pyridine (4VP), ammonium peroxodisulfate (APS), and $N, N, N^{\prime}, N^{\prime}$-tetramethyl ethylenediamine (TEMED) were purchased from Aladdin Reagent Company (Shanghai, China). Urease (U1500, type III from Jack Bean, 15000-50000 catalytic $\mathrm{U} / \mathrm{g}$ ) and fluorescein $O$-methacrylate (FM) were purchased from Sigma-Aldrich (St. Louis, MO, USA). All other reagents were purchased from Sinopharm Chemical Reagent Co., Ltd. (Shanghai, China) and used as received without any further purification. Deionized water was used for all the experiments.

Fabrication of $(\mathbf{x}, \mathbf{1 2}-\mathbf{x})$-hydrogels. The hydrogels were fabricated with varied SSNa and 4VP concentrations. FM and carbon black were used to improve the contrast of the hydrogels in aqueous solutions. The typical fabrication procedure for $(6,6)$-hydrogels is as follows: AAm (61.2 mg, $0.86 \mathrm{mmol}), \mathrm{SSNa}(12.6 \mathrm{mg}, 0.06 \mathrm{mmol}), 4 \mathrm{VP}(7.0 \mu \mathrm{L}, 0.06 \mathrm{mmol}), \mathrm{MBAAm}(3.1 \mathrm{mg}$, $0.02 \mathrm{mmol})$, urease $(6.0 \mathrm{mg})$, TEMED $(1.5 \mu \mathrm{L}, 0.01 \mathrm{mmol})$ and $\mathrm{FM}(1.0 \mathrm{mg})$ were dissolved in water $(1.0 \mathrm{~mL})$. After sufficient mixing under ultrasonication, APS $(2.3 \mathrm{mg}, 0.01 \mathrm{mmol})$ was added to the system. The solution turned into gel within $30 \mathrm{~min}$ at room temperature. The gel was washed repeatedly with deionized water. Other $(\mathrm{x}, 12-\mathrm{x})$-hydrogels were fabricated with a 
similar procedure but with different SSNa and 4VP amounts.

Fabrication of sewed/grown hydrogels. The polyacrylamide (PAAm) precursor aqueous solution was prepared by mixing AAm $(61.2 \mathrm{mg}, 0.86 \mathrm{mmol})$, MBAAm $(3.1 \mathrm{mg}, 0.02 \mathrm{mmol})$, urease $(6.0 \mathrm{mg})$, and TEMED $(1.5 \mu \mathrm{L}, 0.01 \mathrm{mmol})$ in $1.0 \mathrm{~mL}$ of deionized water. After sufficient mixing under ultrasonication, the solution was poured into a rectangular Teflon mold. The urease-containing $(0,12)$ - and $(12,0)$-hydrogels were cut into long strips and assembled along the length, and then immersed into the PAAm precursor solution for $30 \mathrm{~min}$. APS (2.3 $\mathrm{mg}, 0.01$ mmol) was then added to the precursor solution. The urease-containing sewed/grown hydrogels were formed within $20 \mathrm{~min}$.

Fabrication of (x,12-x)-DN hydrogels. The double network (DN) hydrogels were fabricated by immersing the single network $(\mathrm{SN})$ hydrogels in the PAAm precursor solutions, following by the addition of APS to initiate the polymerization. The typical fabrication procedure for $(0,12)$-DN hydrogels included the preparation of the PAAm precursor solution, mixing under ultrasonication, and pouring into the Teflon mold as indicated above. The urease-containing $(0,12)$-hydrogel was cut into long strips and immersed in the aqueous solution for $30 \mathrm{~min}$. APS ( $2.3 \mathrm{mg}, 0.01 \mathrm{mmol})$ was then added to the PAAm precursor solution. The $(0,12)$-DN hydrogels were formed within 20 min. Other $(\mathrm{x}, 12-\mathrm{x})$-DN hydrogels were fabricated with the similar procedure but different SSNa and 4VP amounts.

Determination of urease activity. The urease activity was determined by the titration method. Briefly, $1.8 \mathrm{mg}$ of urease was added to the buffer solution $(3.6 \mathrm{~mL})$ containing urea $(30$ $\mathrm{mg} / \mathrm{mL})$ in a test tube at $25^{\circ} \mathrm{C}$, with or without APS $(2.3 \mathrm{mg} / \mathrm{mL})$ and TEMED $(1.5 \mu \mathrm{L} / \mathrm{mL})$, 
$4 \mathrm{VP}(7 \mu \mathrm{L} / \mathrm{mL})$, and SSNa $(2.3 \mathrm{mg} / \mathrm{mL})$. After $30 \mathrm{~min}$, the enzymatic reaction was terminated by adding $30 \mathrm{~mL}$ of $\mathrm{HCl}(0.12 \mathrm{M})$. The solution was transferred to a beaker, and the test tube was washed twice with water ( $5 \mathrm{~mL}$ each time), and the washing water added to the beaker. The $\mathrm{pH}$ of the system was adjusted to 5.0 with $0.1 \mathrm{M} \mathrm{NaOH}$. The blank test was performed by adding $\mathrm{HCl}$ to the solution, followed by adding the same amount of urease. The urease activity (U/g) was calculated with the following equation:

$$
\text { Urease activity }=\frac{0.1 \times 10^{3} \times\left(V_{0}-V\right)}{2 \times 30 \times 1.8 \times 10^{-3}}
$$

where $V_{0}$ and $V$ are the $\mathrm{NaOH}$ volumes $(\mathrm{mL})$ in the blank and sample, respectively.

Determination of kinetic parameters for immobilized urease. The method is similar to the determination of urease activity. Two pieces of $(0,12)$ - and $(6,6)$-hydrogels immobilized with urease were placed into a buffer solution $(3.6 \mathrm{~mL})$ in a test tube at $25^{\circ} \mathrm{C}$. Different amounts of urea molecules were added to initiate the enzymatic reaction. The reaction rate (the change of urea concentration at a certain time period) was determined by the same titration method mentioned above. The results were fitted by using the Michaelis-Menten equation (eq 2) to obtain the maximal velocity $\left(V_{\max }\right)$ and the Michaelis constant $\left(K_{\mathrm{m}}\right)$ :

$$
\text { Reaction rate }=\frac{V_{\max }[S]}{K_{\mathrm{m}}+[S]}
$$

where $[S]$ is the concentration of urea.

Determination of swelling ratio. The equilibrium swelling ratio (ESR) of (x,12-x)-hydrogels was measured by the gravimetric method. The ESR value was calculated as follows: 


$$
\mathrm{ESR}=\frac{W_{e}-W_{i}}{W_{i}}
$$

where $W_{\mathrm{e}}$ is the weight of swollen hydrogels at equilibrium in buffer solutions with different $\mathrm{pH}$, and $W_{\mathrm{i}}$ is the weight of initial swollen hydrogels at equilibrium in buffer solutions at $\mathrm{pH} 8$.

Macroscopic nonequilibrium assembly. The hydrogels were cut into cubes (size: $5 \times 5 \times 5$ $\mathrm{mm}^{3}$ ). Four pieces of cubic hydrogels were placed into a container with $1.5 \mathrm{~mL}$ of alkaline buffer. The urea-containing acidic buffer (citric acid/sodium citrate) was then added to the container, and the container was placed on a rotating shaker at $180 \mathrm{rpm}$ to trigger the nonequilibrium assembly.

Characterizations. Fourier transform infrared (FTIR) spectra were obtained using a Fourier transform infrared spectrometer (Tensor II, Bruker, MA, USA). Scanning electron microscopy (SEM) images and energy-dispersive spectroscopy (EDS) mapping images were acquired on a Hitachi SU8010 microscope. Confocal laser scanning microscopy images were collected on a Leica TCS SP8X confocal microscope, and the excitation wavelength was $495 \mathrm{~nm}$. UV-vis spectra were collected on a microplate reader (Spark, Tecan, Switzerland). The optical microscopy image was collected on an optical microscope (MP41, Guangzhou Micro-Shot Technology Co., Ltd.). The adhesion strength between hydrogels and the elastic moduli of the hydrogels were measured using a TMS-Pro texture analyzer (Food Technology Corporation) in tensile stress-strain experiments. The probes for the tensile tests were wedge grips (part number 432-298) and the test speed was $60 \mathrm{~mm} / \mathrm{min}$. The zeta potential of the hydrogel particulates was characterized through the laser particle size and zeta potential analyzer (Zetasizer Nano ZS, UK). Each measurement was performed in triplicates. The hydrogel particulates were fabricated by 
crushing the freeze-dried hydrogels.

\section{RESULTS AND DISCUSSION}

Polymer hydrogels have been widely used as model building blocks for macroscopic assembly studies $^{26,32,35,40}$ due to their excellent deformability and motility of the surface and potential applications in tissue engineering ${ }^{37}$ and stimuli-responsive materials. ${ }^{41}$ To demonstrate the macroscopic nonequilibrium assembly, five kinds of polyelectrolyte hydrogels with different charge proportions were fabricated. Figure 1a shows the fabrication processes of urease-containing $(\mathrm{x}, 12-\mathrm{x})$-hydrogels, which were made through radical copolymerization of AAm, MBAAm, 4VP, and/or SSNa in the presence of urease in water. The reaction was initiated by APS and accelerated by TEMED. The parameters $\mathrm{x}$ and 12-x represented the amounts (mol\%) of 4VP and SSNa monomers in the system, respectively. Among these hydrogels, the negatively charged $(0,12)$-hydrogels were stained with carbon black and the others were stained with a yellow dye (FM) to improve the visibility of the hydrogels. The FTIR spectra in Figure S1 suggest the successful introduction of 4VP and/or SSNa units to the hydrogels, as evidenced by the appearance of peaks at 825 and $1176 \mathrm{~cm}^{-1}$, which were assigned to the out-of-plane $\mathrm{C}-\mathrm{H}$ bending vibration of the p-substituted benzene ring and the $\mathrm{C}-\mathrm{N}$ stretching vibration, respectively. The SEM image in Figure S2 reveals that the urease-containing hydrogels presented a porous network structure. The homogeneous distribution of urease in the hydrogel was demonstrated through the EDS mapping results in Figure S2, where the characteristic elements P 
and $\mathrm{K}$ in urease were uniformly distributed in the hydrogel, along with the $\mathrm{C}, \mathrm{O}, \mathrm{S}$, and $\mathrm{N}$. This was further confirmed through the homogeneously distributed fluorescein isothiocyanate (FITC)-labeled urease in the hydrogel (Figure S3). The urease showed moderately reduced activity in the presence of APS and TEMED, 4VP or SSNa (Figure S4), suggesting that the urease remained active in the polyelectrolyte hydrogels. Urease was stably immobilized in the polyelectrolyte network via multiple supramolecular interactions, and thus no urease was released during the long-time immersion of the urease-containing hydrogels in buffer solutions (Figure S5).

a
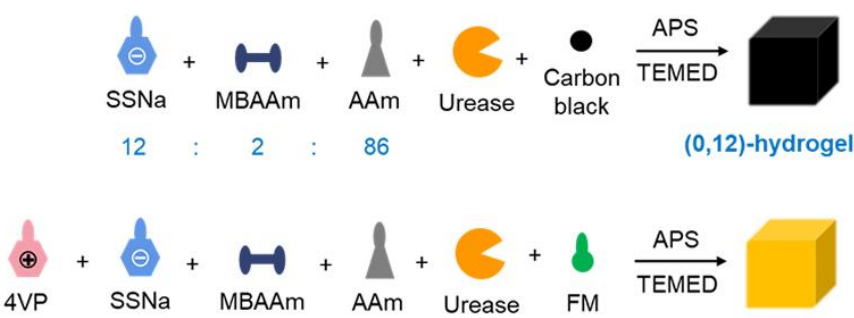

$\begin{array}{lllllll}3 & : & 9 & : & 2 & : & 86 \\ 6 & : & 6 & : & 2 & : & 86 \\ 9 & : & 3 & : & 2 & : & 86\end{array}$

$12: 0: 2: 86$
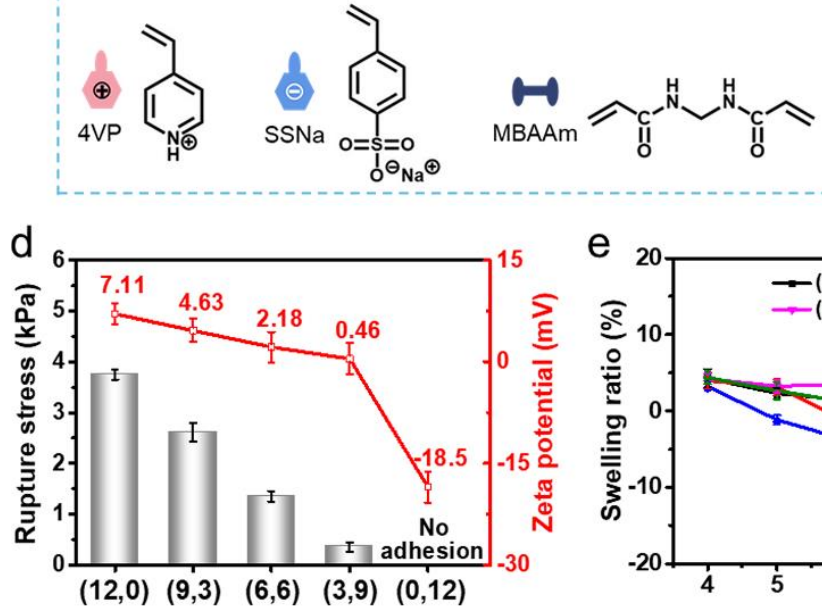

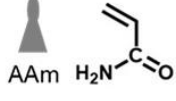

$(3,9)$-hydrogel

$(6,6)$-hydrogel

$(9,3)$-hydroge

$(12,0)$-hydrogel
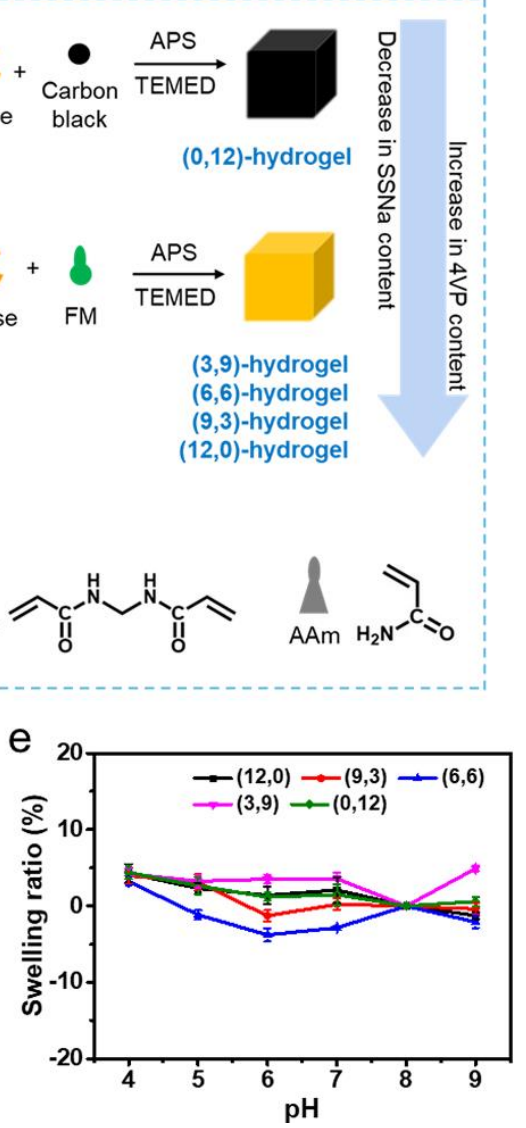

b
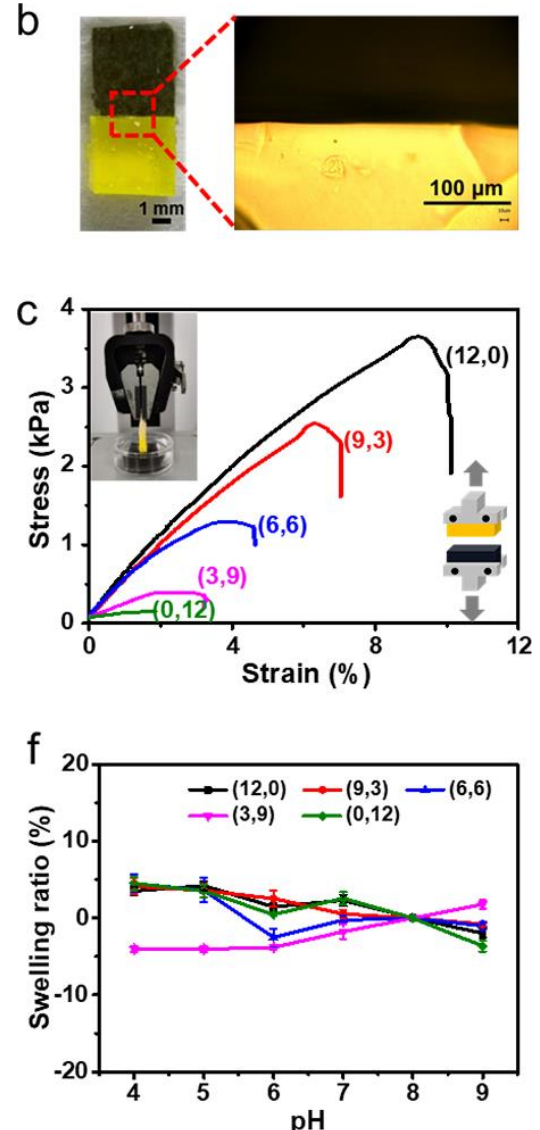

Figure 1. Fabrication of polyelectrolyte hydrogels and measurement of the interactions between oppositely charged hydrogels. (a) Schematic illustrating the synthesis of (x,12-x)-hydrogels. The 
parameters $\mathrm{x}$ and 12-x represent the amounts (mol \%) of 4VP and SSNa monomers used in the hydrogels, respectively. (b) Photograph and microscopic image of combined $(0,12)$ - and $(12,0)$-hydrogels. (c) Typical stress-strain curves of the joined surfaces between $(0,12)$-hydrogels and different hydrogels (contact area: $10 \mathrm{~mm} \times 5 \mathrm{~mm}$ ) at $\mathrm{pH} 3.5$. The insets show the device and principle of the experiment. (d) Rupture stresses between $(0,12)$-hydrogels and different hydrogels and zeta potentials of different hydrogel particulates at $\mathrm{pH} 3.5$. (e, f) Swelling ratios of $(\mathrm{x}, 12-\mathrm{x})$-hydrogels after immersion in buffer solutions with different $\mathrm{pH}$ in the absence (e) and presence (f) of urea $(50 \mathrm{mg} / \mathrm{mL})$ for $400 \mathrm{~min}$.

The 4VP monomer $(\mathrm{pKa}=5.62)$ contains a tertiary amine group which is protonated under acidic conditions, and thus its homopolymer or copolymer has $\mathrm{pH}$ responsiveness. ${ }^{42}$ Accordingly, (x,12-x)-hydrogels $(\mathrm{x}=12,9,6$, or 3$)$ containing 4VP units are $\mathrm{pH}$-sensitive, and present electrostatic interactions with negatively charged $(0,12)$-hydrogels under acidic $\mathrm{pH}$. Figure $1 \mathrm{~b}$ shows the photograph and microscopic image of acidified $(0,12)$ - and (12,0)-hydrogels, which exhibit tight contact at the interacting hydrogel interface. The adhesion strength between $(0,12)-$ and (x,12-x)-hydrogels $(\mathrm{x}=12,9,6,3$, or 0$)$ in the acid buffer $(\mathrm{pH} 3.5)$ was investigated with a texture analyzer. Figure 1c demonstrates the typical procedure for the stress-strain measurements and the tensile curves for the joined surfaces between $(0,12)$-hydrogels and different hydrogels. The combined hydrogels were pulled toward opposite positions, and the rupture stress was obtained from the stress-strain curve as the maximal stretching force divided by the surface-to-surface area. Figure 1d shows that the rupture stress for the joined surface between $(0,12)$ - and $(\mathrm{x}, 12-\mathrm{x})$-hydrogels $(\mathrm{x}=12,9,6,3$, or 0$)$ significantly decreased with decreasing 4VP content at $\mathrm{pH} 3.5$, which was attributed to the decreased zeta potential of the hydrogel particulates, along with the gradually decreased electrostatic attraction. In addition, we compared the adhesion strength between $(0,12)$ - and $(12,0)$-hydrogels in either acidic or alkaline buffers 
and found that there were strong interactions between the hydrogels at acidic $\mathrm{pH}$ but no interactions at basic $\mathrm{pH}$ (Figure S6). These results demonstrate that the interactions between polyelectrolyte hydrogels can be precisely controlled by adjusting the $\mathrm{pH}$ of the system and the contents of the 4VP cation and SSNa anion moieties in the hydrogels. Next, the swelling ratios of (x,12-x)-hydrogels were investigated by immersing the hydrogels in buffer solutions with different $\mathrm{pH}$ in the absence and presence of urea $(50 \mathrm{mg} / \mathrm{mL})$ for $400 \mathrm{~min}$. The results in Figure 1e,f indicate that the hydrogels exhibited negligible swelling or deswelling ratios after long-time immersion in various aqueous mediums, which are conducive to accurately investigating the nonequilibrium assembly behaviors of hydrogels.

The autocatalytic urea-urease clock reaction has been widely used to temporally control the pH of the systems. ${ }^{43-46}$ Using this enzymatic reaction, we previously balanced the kinetic stability and healing ability of acylhydrazone-based polymer hydrogels. ${ }^{24,25}$ Here the reaction was adopted to regulate the nonequilibrium assembly between oppositely charged hydrogels. We found that the number of the hydrogels $(4,6$, or 8$)$ used for assembly and the location of urease (immobilized in the hydrogels or dispersed in the solutions) slightly affected the macroscopic nonequilibrium assembly (Figures S7, S8). To simplify the experimental condition, we chose four building blocks with immobilized urease for further studies. We investigated the macroscopic nonequilibrium assembly between $(0,12)$ - and $(\mathrm{x}, 12$ - $\mathrm{x})$-hydrogels $(\mathrm{x}=12,9,6,3$, or 0) in the solutions with a constant urea concentration of $50 \mathrm{mg} / \mathrm{mL}$. The conceptual scheme of chemically fueled macroscopic nonequilibrium assembly is displayed in Figure $2 \mathrm{a}$. Figure $2 \mathrm{~b}$ depicts that $(0,12)$ - and $(\mathrm{x}, 12-\mathrm{x})$-hydrogels $(\mathrm{x}=12,9,6$, or 3$)$ did not assemble when shaken in 
an alkaline buffer $(\mathrm{pH} 8)$ and assembled to form irregular-shaped aggregates within 2 min when acidic urea solutions were added to the systems. By contrast, $(0,12)$ - and $(0,12)$-hydrogels could not assemble due to the electrostatic repulsion. The time for disassembly decreased with decreasing 4VP content in the hydrogels (Figure $2 b, c)$, which corresponded to the adhesion strength of the joined surfaces between hydrogels. The whole nonequilibrium assembly processes of the hydrogels with different combinations are displayed in Figures S9-S13. When the acidic urea solutions were added, a remarkable acceleration of the $\mathrm{pH}$ decline followed by a slow $\mathrm{pH}$ recovery was observed in all the groups (Figure $2 \mathrm{~d}$ ). The ambient $\mathrm{pH}$ dropped to as low as $\sim 3.8$ and the final $\mathrm{pH}$ of the solutions used for macroscopic nonequilibrium assembly reached $\sim 9.0$ as the urease-regulated catalytic reaction progressed. The zeta potential measurements were conducted to record the time-dependent zeta potential variations of $(\mathrm{x}, 12-\mathrm{x})$-hydrogel particulates $(\mathrm{x}=12,9,6,3$, or 0$)$ after fuel addition. Figure 2e shows that the zeta potential of $(0,12)$-hydrogel particulates without 4VP functionalities generally maintained a constant negative value during the measurement, while the zeta potential of $(x, 12-x)$-hydrogel particulates $(\mathrm{x}=12,9,6$, or 3$)$ generally decreased over time after fuel addition due to the increase in the $\mathrm{pH}$ of the system. Notably, the zeta potentials of $(x, 12-x)$-hydrogels $(x=12,9,6$, or 3$)$ were positive at the beginning but became negative at the end (Figure 2e), suggesting the possibility to perform the switching between electrostatic attraction and repulsion using the urea-urease clock reaction to temporally control the $\mathrm{pH}$ of the system. 
a

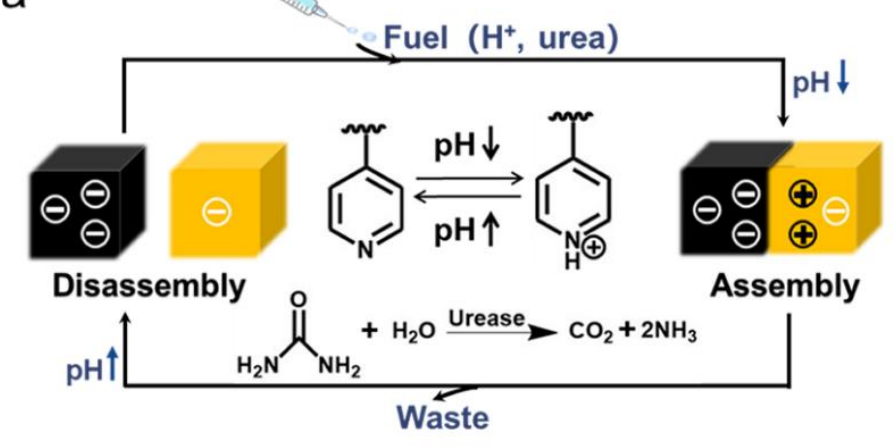

b

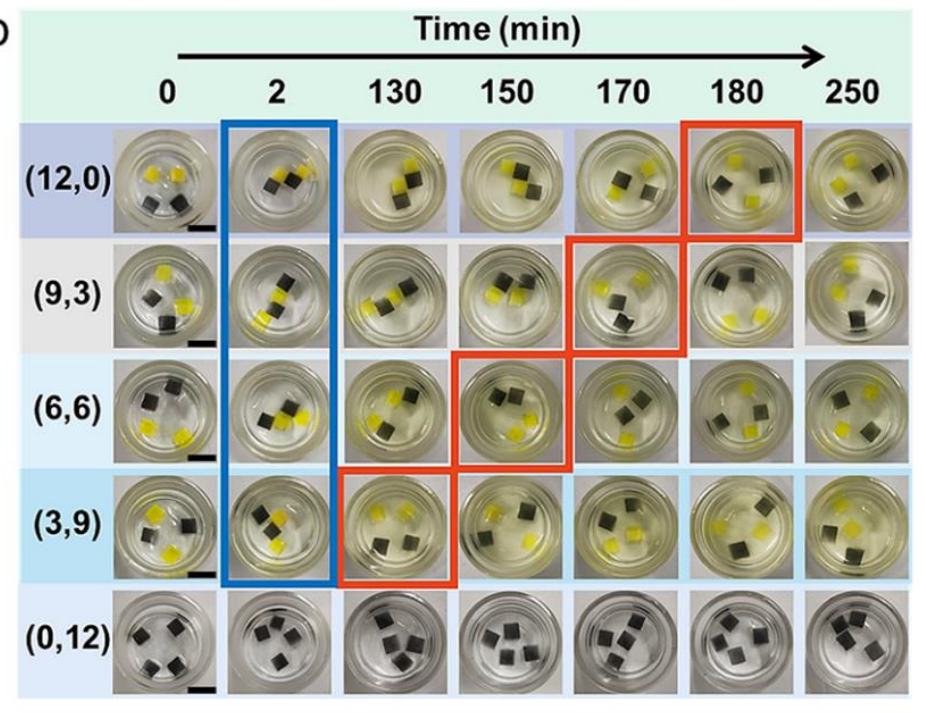

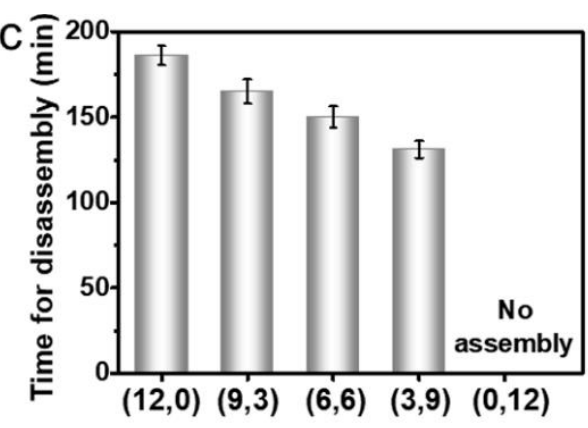

d
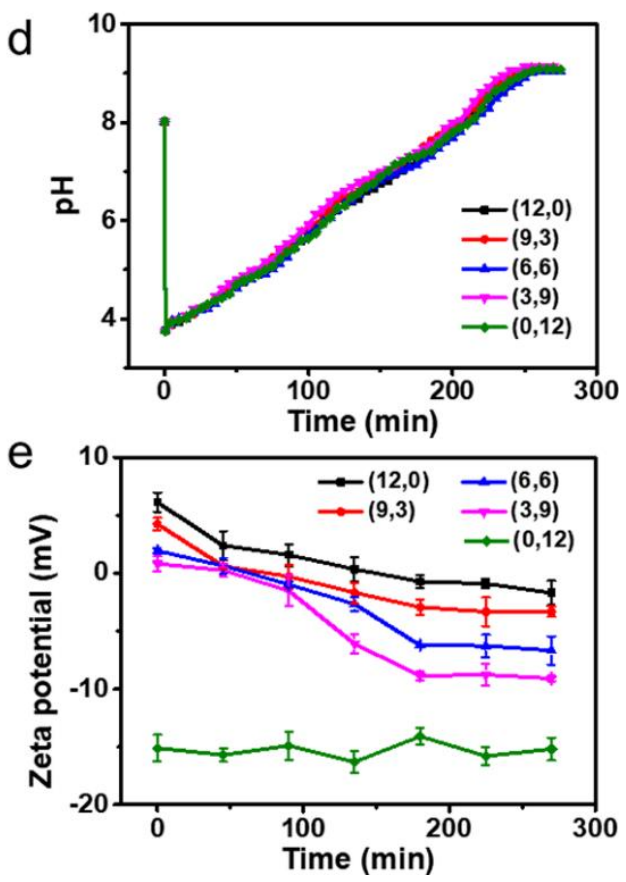

Figure 2. Macroscopic nonequilibrium supramolecular assembly for different hydrogels. (a) Schematic representation of the nonequilibrium supramolecular assembly of macroscopic building blocks. (b) Photographs showing the nonequilibrium supramolecular assembly between $(0,12)$-hydrogels and different hydrogels. Scale bars: $1 \mathrm{~cm}$. The blue and red boxes indicate the starting points for assembly and disassembly, respectively. (c) Time for disassembly in the systems containing $(0,12)$-hydrogels and different hydrogels. (d) Time-dependent $\mathrm{pH}$ variations for the solutions with $(0,12)$-hydrogels and different hydrogels immersed. (e) Time-dependent zeta potential variation for different hydrogel particulates.

Next, we investigated the effect of urea concentrations on assembly-disassembly kinetics using the combination of $(0,12)$ - and $(6,6)$-hydrogels as an example. Figure $3 a$ shows the nonequilibrium assembly processes of $(0,12)$ - and $(6,6)$-hydrogels in the solutions with urea concentrations from 0 to $50 \mathrm{mg} / \mathrm{mL}$. The detailed nonequilibrium assembly processes are 
presented in Figures S14-S19. The hydrogels did not assemble in the initial alkaline buffers (pH 8), while after addition of the acidic urea solutions, the $\mathrm{pH}$ of the system significantly declined to $\sim 3.8$ and the hydrogels assembled after shaking for 2 min (Figure 3a). No disassembly was observed in the group without urea addition, and the time for disassembly in other groups decreased with the increasing urea concentration (Figure 3b). We further determined the enzymatic reaction rate for the immobilized urease by the titration method (details can be found in the Supporting Information) and found that the reaction rate increased as the urea concentration increased below $60 \mathrm{mg} / \mathrm{mL}$ (Figure 3c). The data was fitted by using the Michaelis-Menten equation ${ }^{45}$, which yielded a maximal velocity $\left(V_{\max }\right)$ of $7.6 \pm 0.7 \mathrm{mM} / \mathrm{min}$ and a Michaelis constant $\left(K_{\mathrm{m}}\right)$ of $639.4 \pm 141.1 \mathrm{mM}$. These kinetic parameters are larger than those for free urease,${ }^{47}$ indicating the reduced enzyme activity after being immobilized in the hydrogels, which is in reasonable agreement with the results in Figure S4. The results in Figure 3c indicate that the selected urea concentrations are within an adjustable range, which can well explain the phenomena in Figure 3a,b. The reversibility of the macroscopic nonequilibrium assembly was further validated. Figure S20 shows five cycles of repeated nonequilibrium supramolecular assembly processes of $(0,12)$ - and $(6,6)$-hydrogels. With repeated additions of acidic urea solutions, the $\mathrm{pH}$ of the system periodically switched between 8 and 3.8 (Figure S21), implying that the electrostatic interaction was the driving force for the repeated macroscopic nonequilibrium supramolecular assembly. 

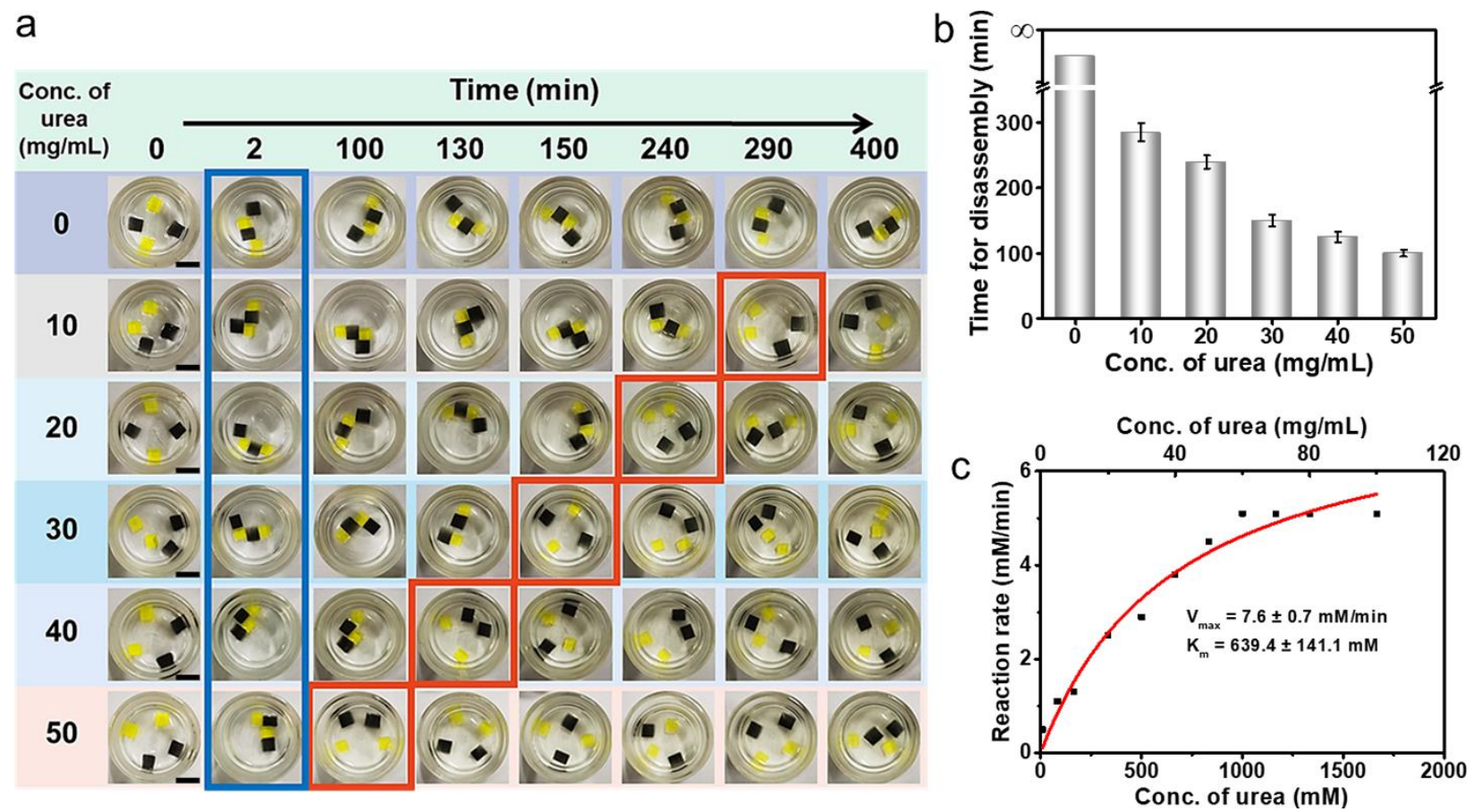

Figure 3. Macroscopic nonequilibrium supramolecular assembly between $(0,12)$ - and $(6,6)$-hydrogels in the solutions with different urea concentrations. (a) Photographs showing the nonequilibrium supramolecular assembly processes. Scale bars: $1 \mathrm{~cm}$. The blue and red boxes indicate the starting points for assembly and disassembly, respectively. (b) Time for disassembly in the systems with different urea concentrations. (c) The Michaelis-Menten curve describing the influence of urea concentration on the enzymatic reaction rate.

After careful investigation on nonequilibrium assembly of macroscopic hydrogels, we focused on precise macroscopic nonequilibrium assembly with hydrogel sewing assistance. ${ }^{48,49}$ Four-layered building blocks containing complementary DN hydrogels were fabricated via an ordered hydrogel sewing/growing method (Figure 4a). The top and bottom layers of the building blocks were non-charged PAAm hydrogels, and the middle two layers were $(0,12)$-DN and $(12,0)$-DN hydrogels. After addition of acidic urea solutions to the system for 5 min, the building blocks assembled precisely into an oriented cuboid (Figure 4b, Video 1) because of the specific binding between the positively and negatively charged sites. As the urea-urease clock reaction 
progressed, the aggregate disassembled once the energy was depleted. By analyzing the structure of the building blocks, we noticed that, in principle, there were diverse assembly shapes and imprecise assembly might occur during the assembly process. However, the experimental results show that the orderly sewed/grown hydrogels can assemble only into a well-defined straight shape (Figure 4b). We hypothesize that this phenomenon is caused by the increased elastic modulus of the charged hydrogels after sewing, which decreases the electrostatic attraction between hydrogels and makes the macroscopic assemblies with small contact areas easy to dissociate (Figure S22). To test this hypothesis, we measured the elastic moduli of (x,12-x)- and $(\mathrm{x}, 12-\mathrm{x})$-DN hydrogels $(\mathrm{x}=12,9,6,3$, or 0$)$. Figure $4 \mathrm{c}$ shows that for the hydrogels with the same charge ratio, the elastic moduli of $\mathrm{SN}$ hydrogels were lower than those for the DN hydrogels. Among the groups with different contents of 4VP and SSNa functionalities, the largest change in elastic modulus occurred in the group containing $(12,0)$ - and $(12,0)$-DN hydrogels, in which the elastic modulus increased from $\sim 6$ to $\sim 12 \mathrm{kPa}$. The adhesive strength between $(0,12)$-DN and $(x, 12-x)$-DN hydrogels $(x=12,9$, or 6$)$ was then measured. The stress-strain curves shown in Figure $4 \mathrm{~d}$ indicate that $(0,12)$-DN hydrogels assemble with $(12,0)$-DN hydrogels, but do not efficiently assemble with $(9,3)$-DN and (6,6)-DN hydrogels. When compared to the adhesive strength for the SN hydrogel systems in Figure 1c, the binding strength between the DN hydrogels greatly reduced because of the increased elastic modulus of the hydrogels. According to the conclusions drawn by Shi and coworkers, the macroscopic supramolecular assembly probability declines with increasing substrate rigidity (elastic modulus) under similar surface chemistry and once the modulus reaches the boundary value, no assembly 
between the hydrogels occurs. ${ }^{50}$ Therefore, as the elastic modulus of the sewed hydrogels increases, the contact area between the sewed hydrogels must be large enough to enhance the interfacial interactions for maintaining the stable assembly under shaking. If the contact area is too small, the imprecise assemblies will dissociate due to the weak adhesive strength. This is the major factor in achieving the precise nonequilibrium supramolecular assembly of the orderly sewed/grown hydrogels.

a Four-layer structure

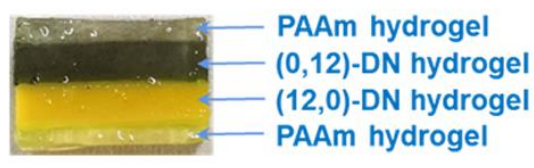

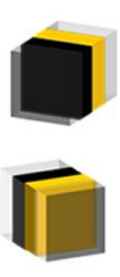

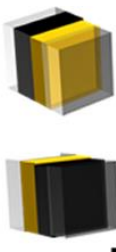

Fuel

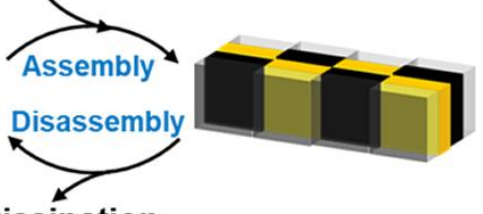

Dissipation

b

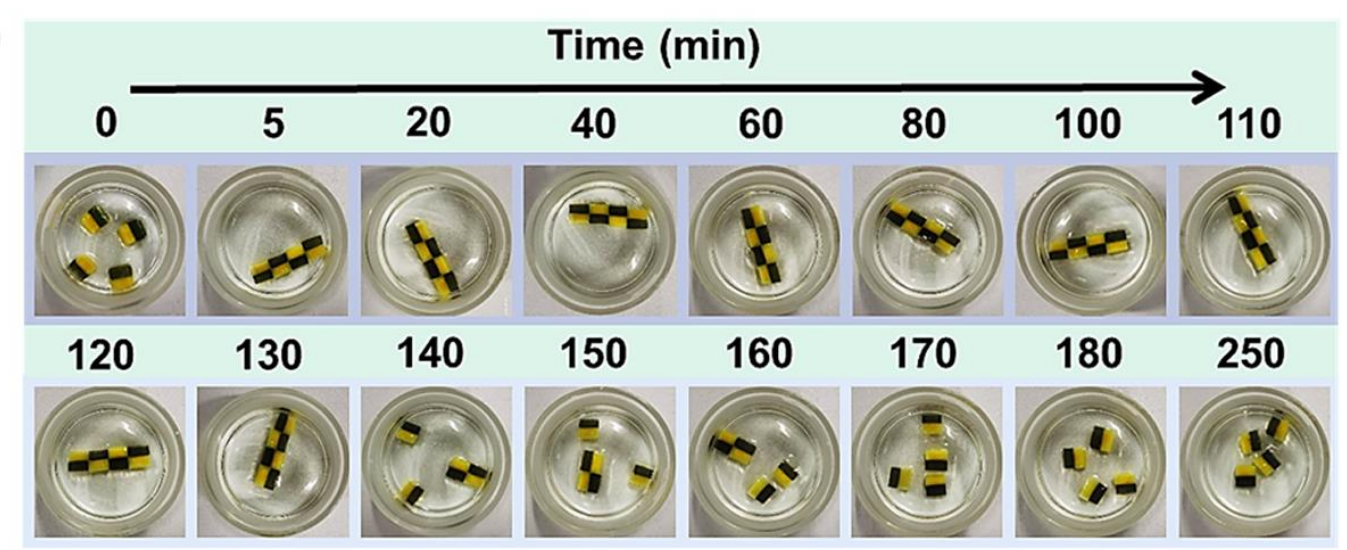

$\mathrm{C}$
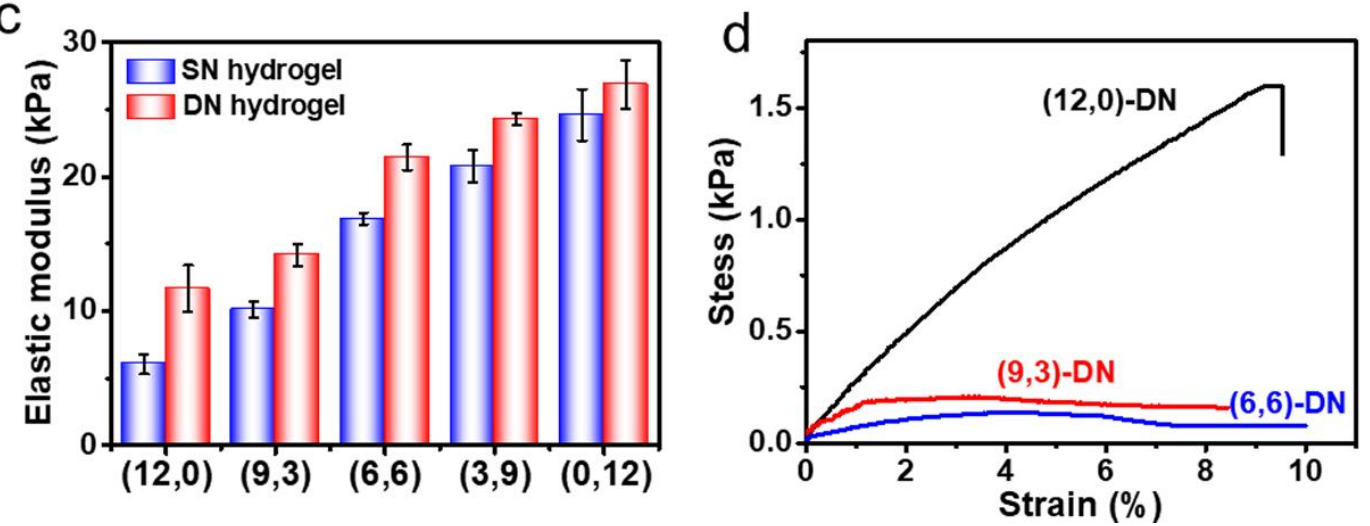

Figure 4. Precise macroscopic nonequilibrium supramolecular assembly. (a) Schematic 
illustration of the structure and transient assembly process of sewed/grown hydrogels. (b) Photographs showing the macroscopic nonequilibrium supramolecular assembly process of sewed/grown hydrogels. Scale bar: $1 \mathrm{~cm}$. (c) Elastic moduli of $(\mathrm{x}, 12-\mathrm{x})-\mathrm{SN}$ and $(\mathrm{x}, 12-\mathrm{x})-\mathrm{DN}$ hydrogels. (d) Typical stress-strain curves of the joined surfaces between $(0,12)$-DN hydrogels and different DN hydrogels at $\mathrm{pH} 3.5$.

To explore the potential application of the macroscopic nonequilibrium supramolecular assembly, orderly sewed/grown hydrogels with specific shapes (Figure 5a,b) that could form a transient container for timed release were fabricated. Figure 5a shows the working principle and process of the transient macroscopic supramolecular device. Notably, human intervention was used to facilitate the formation of a well-defined macroscopic container. After formation of the container upon fuel addition, a cargo with a sharp-bottom holder as shown on the left of Figure $5 \mathrm{~b}$ was placed into the center of the container. As the urease-containing hydrogels were exposed to the urea solution, urea started to hydrolyze and increase the $\mathrm{pH}$ of the system via ammonia production. When the $\mathrm{pH}$ of the system was higher than the $\mathrm{pKa}$ of pyridine groups, the transient container started to dissociate. This led to the collapse of the sharp-bottom holder and, therefore, the cargo was released into the solution. Figure $5 \mathrm{~b}$ shows the entire process of timed release in the solution with a urea concentration of $50 \mathrm{mg} / \mathrm{mL}$. When the transient container disassembled at a time point of $125 \mathrm{~min}$, a model cargo (i.e., an effervescent tablet) dropped into the aqueous solution and generated bubbles. This fact indicated the successful timed release via macroscopic nonequilibrium supramolecular assembly. Moreover, we can further regulate the cargo release time by adjusting the urea concentration. Five different conditions were used to study the influence of urea concentrations on the lifetime of the transient macroscopic supramolecular 
assemblies. We found that the lifetime decreased with increasing urea concentration (Figure 5c), suggesting that the release time can be controlled by adjusting the urea concentration and both were negatively correlated. Figure $5 \mathrm{~d}$ shows the influence of urea concentration on the $\mathrm{pH}$ of the system. The higher urea concentration led to a faster $\mathrm{pH}$ increasing rate, resulting in a shorter lifetime of the transient macroscopic supramolecular device. The controllable and programmable lifetime of the transient supramolecular assemblies is particularly attractive for timed feeding in closed analytical systems. In addition to timed release, the precise nonequilibrium supramolecular assembly of macroscopic building blocks is also promising to be applied for mass transfer when combined with fluid environments. 


\section{a (0,12)-DN hydrogel PAAm hydrogel}

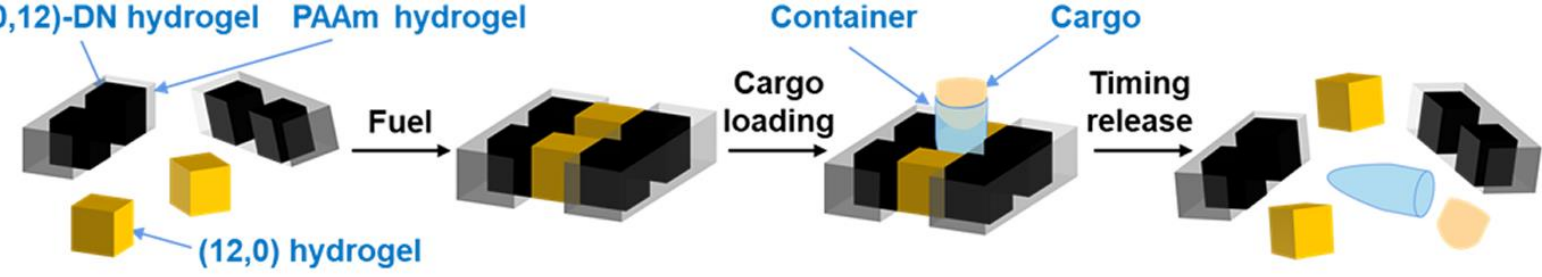

b

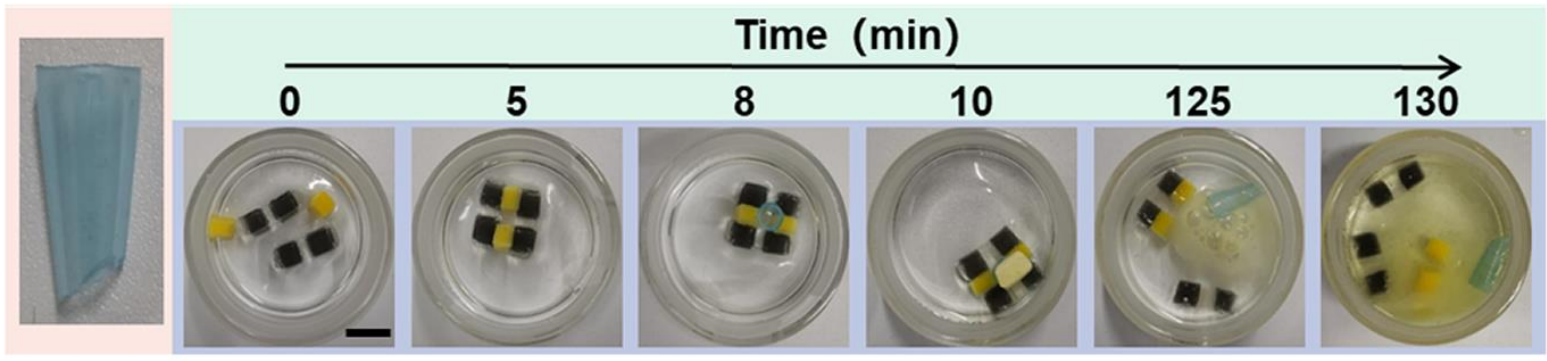

C

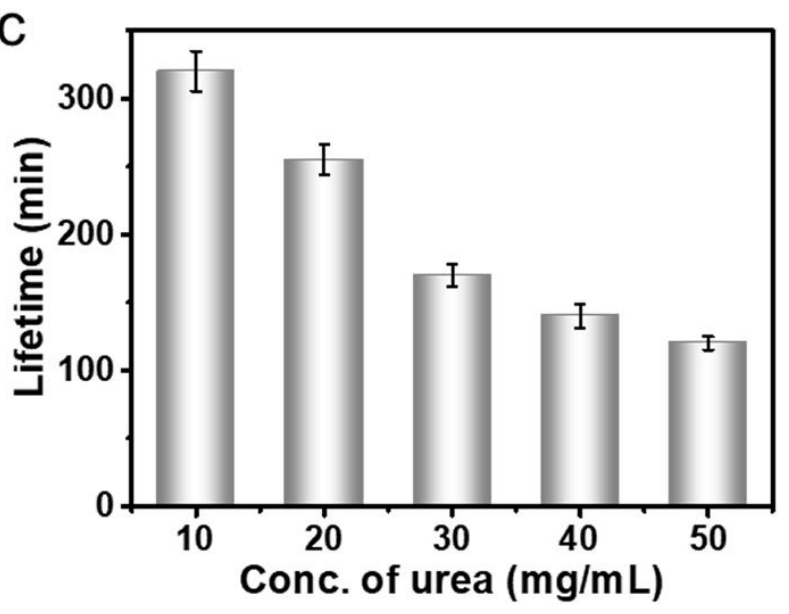

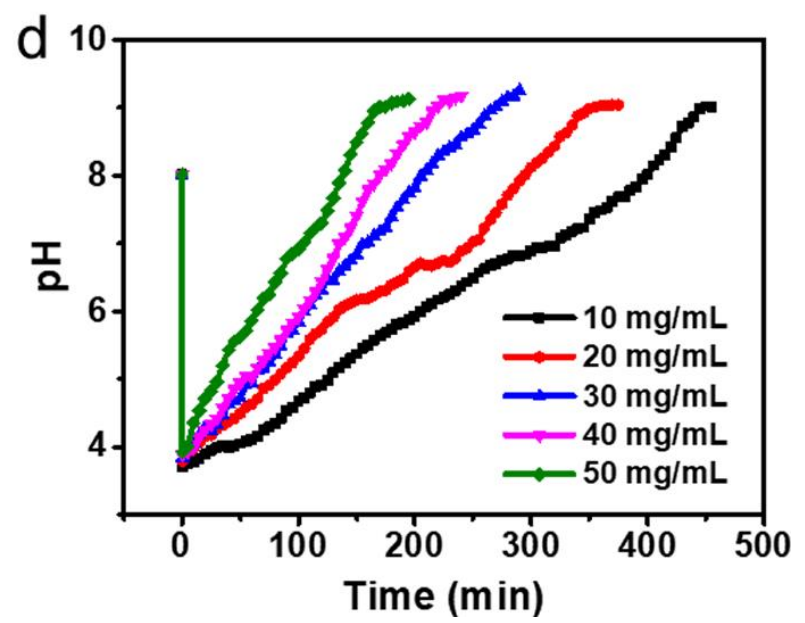

Figure 5. Construction of a transient macroscopic supramolecular device for timed release. (a) Schematic illustration of the timed cargo release using a transient macroscopic supramolecular device. (b) Photographs showing the timed-release process using a transient macroscopic supramolecular device. Scale bar: $1 \mathrm{~cm}$. (c) Lifetimes of the transient macroscopic assemblies fueled by acidic urea solutions with different urea concentrations. (d) Influence of urea concentrations on the $\mathrm{pH}$ of the system.

\section{CONCLUSIONS}

In this study, we present the first example of precise nonequilibrium supramolecular assembly of macroscopic building blocks using an enzyme-mediated, $\mathrm{pH}$-sensitive polyelectrolyte hydrogel 
system. This contribution fills the gap in the field of macroscopic nonequilibrium supramolecular assembly and overcomes the technical difficulty in building block design for achieving precise macroscopic supramolecular assembly. The properties of the transient macroscopic assemblies based on temporally programmed electrostatic interactions, including their stability and lifetime, can be precisely controlled by adjusting the hydrogel synthesis parameters and chemical fuel compositions. More importantly, we developed an ordered sewing/growing method to fabricate well-defined hydrogels with multilayered structures and complementary interactions through in situ polymerization of crosslinked polymers interpenetrated in and extended outside the original hydrogel networks, which plays an important role in achieving the precise nonequilibrium supramolecular assembly of macroscopic building blocks. The principle and methods presented in this study cannot only solve the problems of control over dynamic and precise macroscopic supramolecular assembly but also promote the creation of self-adaptive chemical systems for potential applications such as controlled release and mass transfer.

\section{ASSOCIATED CONTENT}

\section{Supporting Information}

The Supporting Information is available free of charge.

FTIR spectra, SEM and EDS images, confocal laser scanning microscopy images, urease activity studies, UV-vis spectra, measurements of the interfacial interactions, macroscopic nonequilibrium assembly processes, repeated macroscopic nonequilibrium assembly processes, and possible models for macroscopic assembly of sewed/grown hydrogels (PDF) 
Video 1 as described in the text (MP4)

\section{AUTHOR INFORMATION}

\section{Corresponding Author}

Xu Wang - Shandong University, Jinan, China; orcid.org/0000-0002-6646-0515; Email:

wangxu@sdu.edu.cn

Notes

The authors declare no competing financial interest.

\section{ACKNOWLEDGMETNS}

This work was supported by the National Natural Science Foundation of China (Grant No. 21975145).

\section{REFERENCES}

(1) Roduner, E.; Radhakrishnan, S. G., In Command of Non-Equilibrium. Chem. Soc. Rev. 2016, $45,2768-2784$.

(2) Sorrenti, A.; Leira-Iglesias, J.; Sato, A.; Hermans, T. M., Non-Equilibrium Steady States in Supramolecular Polymerization. Nat. Commun. 2017, 8, 15899.

(3) Merindol, R.; Walther, A., Materials Learning from Life: Concepts for Active, Adaptive and Autonomous Molecular Systems. Chem. Soc. Rev. 2017, 46, 5588-5619.

(4) Das, K.; Gabrielli, L.; Prins, L. J., Chemically Fueled Self-Assembly in Biology and 
Chemistry. Angew. Chem. Int. Ed. 2021, 60, 20120-20143.

(5) van Esch, J. H.; Klajn, R.; Otto, S., Chemical Systems out of Equilibrium. Chem. Soc. Rev. 2017, 46, 5474-5475.

(6) Ragazzon, G.; Prins, L. J., Energy Consumption in Chemical Fuel-Driven Self-Assembly. Nat. Nanotechnol. 2018, 13, 882-889.

(7) Singh, N.; Formon, G. J. M.; De Piccoli, S.; Hermans, T. M., Devising Synthetic Reaction Cycles for Dissipative Nonequilibrium Self-Assembly. Adv. Mater. 2020, 32, 1906834.

(8) Gregorio, C. C.; Antin, P. B., To the Heart of Myofibril Assembly. Trends Cell Biol. 2000, 10, $355-362$

(9) Whitesides, G. M.; Grzybowski, B., Self-Assembly at All Scales. Science 2002, 295, 2418-2421.

(10)Boekhoven, J.; Brizard, A. M.; Kowlgi, K. N.; Koper, G. J. M.; Eelkema, R.; van Esch, J. H., Dissipative Self-Assembly of a Molecular Gelator by Using a Chemical Fuel. Angew. Chem. Int. Ed. 2010, 49, 4825-4828.

(11)Panja, S.; Boháčová, K.; Dietrich, B.; Adams, D. J., Programming Properties of Transient Hydrogels by an Enzymatic Reaction. Nanoscale 2020, 12, 12840-12848.

(12)Hossain, M. M.; Atkinson, J. L.; Hartley, C. S., Dissipative Assembly of Macrocycles Comprising Multiple Transient Bonds. Angew. Chem. Int. Ed. 2020, 59, 13807-13813.

(13)Ghosh, A.; Paul, I.; Schmittel, M., Multitasking with Chemical Fuel: Dissipative Formation of a Pseudorotaxane Rotor from Five Distinct Components. J. Am. Chem. Soc. 2021, 143, 5319-5323. 
(14)Jain, A.; Dhiman, S.; Dhayani, A.; Vemula, P. K.; George, S. J., Chemical Fuel-Driven Living and Transient Supramolecular Polymerization. Nat. Commun. 2019, 10, 450.

(15)Boekhoven, J.; Hendriksen, W. E.; Koper, G. J. M.; Eelkema, R.; van Esch, J. H., Transient Assembly of Active Materials Fueled by a Chemical Reaction. Science 2015, 249, 1075-1079.

(16)Kodura, D.; Houck, H. A.; Bloesser, F. R.; Goldmann, A. S.; Du Prez, F. E.; Frisch, H.; Barner-Kowollik, C., Light-Fueled Dynamic Covalent Crosslinking of Single Polymer Chains in Non-Equilibrium States. Chem. Sci. 2020, 12, 1302-1310.

(17)Pappas, C. G.; Sasselli, I. R.; Ulijn, R. V., Biocatalytic Pathway Selection in Transient Tripeptide Nanostructures. Angew. Chem. Int. Ed. 2015, 54, 8119-8123.

(18)Dai, K.; Fores, J. R.; Wanzke, C.; Winkeljann, B.; Bergmann, A. M.; Lieleg, O.; Boekhoven, J., Regulating Chemically Fueled Peptide Assemblies by Molecular Design. J. Am. Chem. Soc. 2020, 142, 14142-14149.

(19)Guo, J.; Zhang, H. Y.; Zhou, Y.; Liu, Y., Light-Controlled Reversible Self-Assembly of Nanorod Suprastructures. Chem. Commun. 2017, 53, 6089-6092.

(20)Samanta, D.; Klajn, R., Aqueous Light-Controlled Self-Assembly of Nanoparticles. Adv. Optical Mater. 2016, 4, 1373-1377.

(21)Leffler, V. B.; Ehlert, S.; Förster, B.; Dulle, M.; Förster, S., 3D-Positioning of Nanoparticles in High-Curvature Block Copolymer Domains. Angew. Chem. Int. Ed. 2021, 60, 17539-17546.

(22)Schwarz, P. S.; Tebcharani, L.; Heger, J. E.; Müller-Buschbaum, P.; Boekhoven, J., Chemically Fueled Materials with a Self-Immolative Mechanism: Transient Materials with a Fast On/Off Response. Chem. Sci. 2021, 12, 9969-9976. 
(23)Heuser, T.; Weyandt, E.; Walther, A., Biocatalytic Feedback-Driven Temporal Programming of Self-Regulating Peptide Hydrogels. Angew. Chem. Int. Ed. 2015, 54, 13258-13262.

(24)Zhong, Y.; Li, P.; Hao, J.; Wang, X., Bioinspired Self-Healing of Kinetically Inert Hydrogels Mediated by Chemical Nutrient Supply. ACS Appl. Mater. Interfaces 2020, 12, 6471-6478.

(25)Li, P.; Zhong, Y.; Wang, X.; Hao, J., Enzyme-Regulated Healable Polymeric Hydrogels. ACS Cent. Sci. 2020, 6, 1507-1522.

(26)Ju, G.; Guo, F.; Zhang, Q.; Kuehne, A. J. C.; Cui, S.; Cheng, M.; Shi, F., Self-Correction Strategy for Precise, Massive, and Parallel Macroscopic Supramolecular Assembly. Adv. Mater. 2017, 29, 1702444.

(27)Loget, G.; Kuhn, A., Electric Field-Induced Chemical Locomotion of Conducting Objects. Nat. Commun. 2011, 2, 535.

(28)Gao, W.; Sattayasamitsathit, S.; Manesh, K. M.; Weihs, D.; Wang, J., Magnetically Powered Flexible Metal Nanowire Motors. J. Am. Chem. Soc. 2010, 132, 14403-14405.

(29)Sekine, T.; Kakuta, T.; Nakamura, T.; Kobayashi, Y.; Takashima, Y.; Harada, A., A Macroscopic Reaction: Direct Covalent Bond Formation between Materials Using a Suzuki-Miyaura Cross-Coupling Reaction. Sci. Rep. 2014, 4, 6348.

(30)Harada, A.; Kobayashi, R.; Takashima, Y.; Hashidzume, A.; Yamaguchi, H., Macroscopic Self-Assembly through Molecular Recognition. Nat. Chem. 2011, 3, 34-37.

(31)Nakahata, M.; Takashima, Y.; Yamaguchi, H.; Harada, A., Redox-Responsive Self-Healing Materials Formed from Host-Guest Polymers. Nat. Commun. 2011, 2, 511.

(32)Li, J.; Xu, Z.; Xiao, Y.; Gao, G.; Chen, J.; Yin, J.; Fu, J., Macroscopic Assembly of 
Oppositely Charged Polyelectrolyte Hydrogels. J. Mater. Chem. B 2018, 6, 257-264.

(33)Cedano-Serrano, F. J.; Sidoli, U.; Synytska, A.; Tran, Y.; Hourdet, D.; Creton, C., From Molecular Electrostatic Interactions and Hydrogel Architecture to Macroscopic Underwater Adherence. Macromolecules 2019, 52, 3852-3862.

(34)Lehn, J. M., Supramolecular Chemistry: Where From? Where To? Chem. Soc. Rev. 2017, 46, 2378-2379.

(35)Nakahata, M.; Takashima, Y.; Harada, A., Redox-Responsive Macroscopic Gel Assembly Based on Discrete Dual Interactions. Angew. Chem. Int. Ed. 2014, 53, 3617-3621.

(36) Mondal, T.; Sakurai, T.; Yoneda, S.; Seki, S.; Ghosh, S., Semiconducting Nanotubes by Intrachain Folding Following Macroscopic Assembly of a Naphthalene-Diimide (Ndi) Appended Polyurethane. Macromolecules 2015, 48, 879-888.

(37)Cheng, M.; Wang, Y.; Yu, L.; Su, H.; Han, W.; Lin, Z.; Li, J.; Hao, H.; Tong, C.; Li, X.; Shi, F., Macroscopic Supramolecular Assembly to Fabricate 3D Ordered Structures: Towards Potential Tissue Scaffolds with Targeted Modification. Adv. Funct. Mater. 2015, 25, 6851-6857. (38)Nakahata, M.; Mori, S.; Takashima, Y.; Hashidzume, A.; Yamaguchi, H.; Harada, A., pHand Sugar-Responsive Gel Assemblies Based on Boronate-Catechol Interactions. ACS Macro Lett. 2014, 3, 337-340.

(39)Zheng, Y.; Hashidzume, A.; Takashima, Y.; Yamaguchi, H.; Harada, A., Temperature-Sensitive Macroscopic Assembly Based on Molecular Recognition. ACS Macro Lett. 2012, 1, 1083-1085.

(40)Hashidzume, A.; Zheng, Y.; Takashima, Y.; Yamaguchi, H.; Harada, A., Macroscopic 
Self-Assembly Based on Molecular Recognition: Effect of Linkage between Aromatics and the Gel Scaffold, Amide Versus Ester. Macromolecules 2013, 46, 1939-1947.

(41) Yan, Y.-H.; Rong, L.-H.; Ge, J.; Tiu, B. D. B.; Cao, P.-F.; Advincula, R. C., Mussel-Inspired Hydrogel Composite with Multi-Stimuli Responsive Behavior. Macromol. Mater. Eng. 2019, 304, 1800720.

(42)Dogu, S.; Kilic, M.; Okay, O., Collapse of Acrylamide-Based Polyampholyte Hydrogels in Water. J. Appl. Polym. Sci. 2009, 113, 1375-1382.

(43)Hao, X.; Yang, K.; Wang, H.; Peng, F.; Yang, H., Biocatalytic Feedback-Controlled Non-Newtonian Fluids. Angew. Chem. Int. Ed. 2020, 59, 4314-4319.

(44)Panja, S.; Adams, D. J., Urea-Urease Reaction in Controlling Properties of Supramolecular Hydrogels: Pros and Cons. Chem. Eur. J. 2021, 27, 8928-8939.

(45)Wrobel, M. M.; Bánsági, T., Jr.; Scott, S. K.; Taylor, A. F.; Bounds, C. O.; Carranza, A.; Pojman, J. A., pH Wave-Front Propagation in the Urea-Urease Reaction. Biophys. J. 2012, 103, 610-615.

(46)Jee, E.; Bánsági, T., Jr.; Taylor, A. F.; Pojman, J. A., Temporal Control of Gelation and Polymerization Fronts Driven by an Autocatalytic Enzyme Reaction. Angew. Chem. Int. Ed. 2016, 55, 2127-2131.

(47)Alatawi, F. S.; Monier, M.; Elsayed, N. H., Amino Functionalization of Carboxymethyl Cellulose for Efficient Immobilization of Urease. Int. J. Biol. Macromol. 2018, 114, 1018-1025.

(48)Tamesue, S.; Endo, T.; Ueno, Y.; Tsurumaki, F., Sewing Hydrogels: Adhesion of Hydrogels Utilizing in Situ Polymerization of Linear Polymers inside Gel Networks. Macromolecules 2019, 
$52,5690-5697$.

(49)Ji, X.; Li, Z.; Hu, Y.; Xie, H.; Wu, W.; Song, F.; Liu, H.; Wang, J.; Jiang, M.; Lam, J. W. Y.;

Tang, B. Z., Bioinspired Hydrogels with Muscle-Like Structure for Aiegen-Guided Selective Self-Healing. CCS Chem. 2021, 3, 1146-1156.

(50)Sun, Y.; Wang, X.; Xiao, M.; Lv, S.; Cheng, M.; Shi, F., Elastic-Modulus-Dependent Macroscopic Supramolecular Assembly of Poly(Dimethylsiloxane) for Understanding Fast Interfacial Adhesion. Langmuir 2021, 37, 4276-4283. 


\section{For Table of Contents use only}
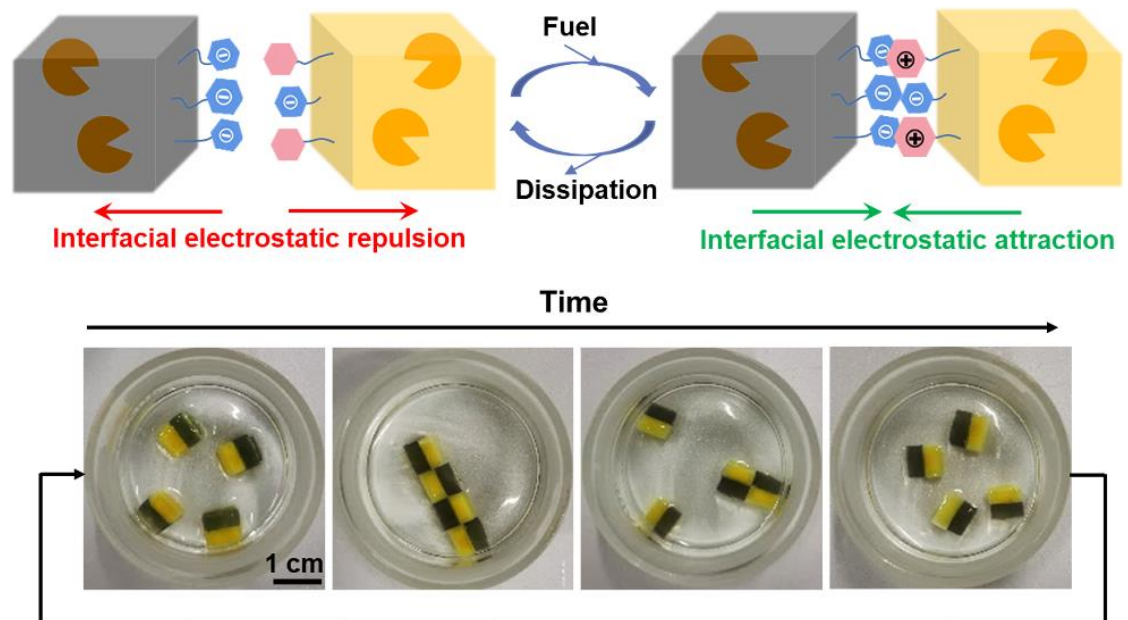

Title: Nonequilibrium Regulation of Interfacial Chemistry for Dissipative Supramolecular Assembly of Macroscopic Hydrogels

Authors: Ting Zhao, Yuyu E, Jiwei Cui, Jingcheng Hao, Xu Wang* 